\title{
Application of Traditional Chinese Medicine Combined Hemodialysis in the Treatment of Acute Paraquat Poisoning
}

\author{
Runhong Wei, 2, a , Xianhui Liu, 2, b and Xiaona Guo ${ }^{3, c}$ \\ ${ }^{1}$ Henan Province Hospital of TCM, 450000, Henan, P. R. China \\ ${ }^{2}$ The Second Affiliated Hospital of Henan University of Traditional Chinese Medicine, 450000, Henan, \\ P. R. China \\ ${ }^{3}$ Huanghe Science and Technology College, Zhengzhou, Henan, P. R. China \\ arunhongwei@163.com, 'bliuxianhui998@sina.com, Ig8509840@163.com
}

Keywords: Paraquat; Poisoning; Blood purification; Clinical treatment

\begin{abstract}
In recent years, with the pesticide Paraquat is widely used in the fields in the event of acute paraquat poisoning are also increasing, but the rescue success rate is not optimistic. Paraquat is highly toxic in contact through the digestive tract, can damage multiple body organs, the highest lung toxicity. Paraquat poisoning is no specific antidote, and high mortality. Blood purification is an effective means to remove the body of toxic substances. This paper analyzes several common blood purification treatment, provided the basis for the clinical treatment of patients with acute paraquat.
\end{abstract}

\section{Introduction}

Paraquat is a highly efficient non-selective contact herbicide spraying effect from rising rapidly after entering the soil rapidly inactivated, no residue in the soil, but also highly toxic to humans and animals. After many experts and scholars at home and abroad long-term study found paraquat poisoning is one of the most common pesticide poisoning due to the use of paraquat more common in developing countries, thus leading to eating Coverage of events that occur frequently in recent years showing a rising trend. Some Chinese statistics derived from hospital emergency departments, paraquat has become after the second organophosphorus pesticide poisoning, pesticide poisoning death of the first type of absolute [1].

With the market Paraquat is widely used after, accompanied by the growing number of PQ poisoning, high mortality PQ poisoning, there is no specific antidote, pesticide use has become a serious clinical problem countries. PQ poisoning can lead to multiple organ damage, and lung damage mainly based. An early manifestation of lung epithelial cell damage, and pulmonary hemorrhage, inflammatory cell infiltration, late can lead to pulmonary fibrosis, respiratory failure caused the death, this is the most PQ poisoning important cause of death. Mechanism of PQ poisoning caused lung injury is not yet entirely clear, the main mechanism of oxygen free radical theory, doctrine enzyme imbalance, loss of mitochondrial theory, in which the oxygen free radical theory is most important. After the PQ poisoning the body to release large amounts of oxygen free radicals, resulting in superoxide dismutase large quantities consumed and the activity was reduced, while the MDA content increased [2]. The main treatment currently rescue PQ poisoning in clinical medicine in the comprehensive treatment (including gastric lavage, catharsis, corticosteroids and immunosuppressive agents, scavenging oxygen free radicals, protecting the organ, etc.) on the basis of, and actively cooperate with effective blood purification treatment. Hemoperfusion (HP), hemoperfusion combined with hemodialysis (HP + HD) are a method of treatment of blood purification, and effective blood purification is a key measure of the current rescue PQ poisoning, blood adsorption technology through the patient's blood accumulated PQ components removed to reduce PQ further damage to the organ so as to achieve the purpose of blood purification. The purpose of the experiment was to investigate the treatment with HP-based blood purification treatment for removal of PQ, PQ to reduce further damage to the organ, which amounted to] the purpose of purification. 


\section{The Pathogenic Mechanism Analysis}

Currently mechanism for paraquat poisoning is still no uniform standard. The more common argument is that it is an electronic receptors, which occurs in the intracellular redox reaction, the reaction produce large amounts of active free radicals, the free radicals can cause lipid peroxidation, cause oxidative damage to cells . In addition, because the lungs alveolar paraquat can produce a strong uptake and accumulation, and therefore, the toxicity of paraquat lung injury and most prominent.

Paraquat has hardly volatile, generally difficult through the gastrointestinal tract, respiratory tract and skin absorption, inhalation poisoning incident unlikely to occur. But easy to paraquat aqueous, if prolonged skin contact with paraquat solution or short exposure to high concentrations of paraquat solution, can cause poisoning. If the skin breakage or scrotum, perineum and other parts exposed to paraquat solution will easily lead to systemic toxicity. After long-term clinical study found that paraquat partially degraded in vivo, swallow after 1-2 days, the poison of the prototype through the kidneys finally excreted in the urine. Poison the prototype passes the tubular kidney damage, acute renal tubular necrosis, but can also cause the body's heart, liver and other organs and nervous system damage. After poisoning can cause lung damage from 1 to 14 days, the early injury of pulmonary congestion and edema in the later becomes widespread pulmonary fibrosis. Pulmonary fibrosis can cause patients have progressive disease and dyspnea, cyanosis, eventually leading to respiratory failure [3].

In summary, the paraquat cause systemic poisoning of the clinical manifestations can be divided into three stages: the first stage, oropharynx, esophagus, stomach, small intestine and other mucosal layer of swelling, edema, ulceration. The second stage, the central area of liver cells damaged, proximal tubular damage, cardiac, skeletal partial necrosis, and some nervous system and pancreas damage. The third stage, the parenchymal cells of the lung damage, pulmonary hemorrhage, edema and leukocyte immersed in the alveoli, pulmonary fibrosis, cell proliferation and severely impaired gas exchange, resulting in blood and tissue hypoxia and death.

\section{Blood Purification Technology}

Blood purification is the use of certain instruments and equipment, the patient's blood leads to the body, through certain procedures rid of certain metabolic waste products or toxic substances, and then the blood brought back processes in the body [4], as shown in Fig. 1. Common blood purification technology hemodialysis, blood filtration, filtration vessels, blood perfusion, replacement therapy, peritoneal dialysis.

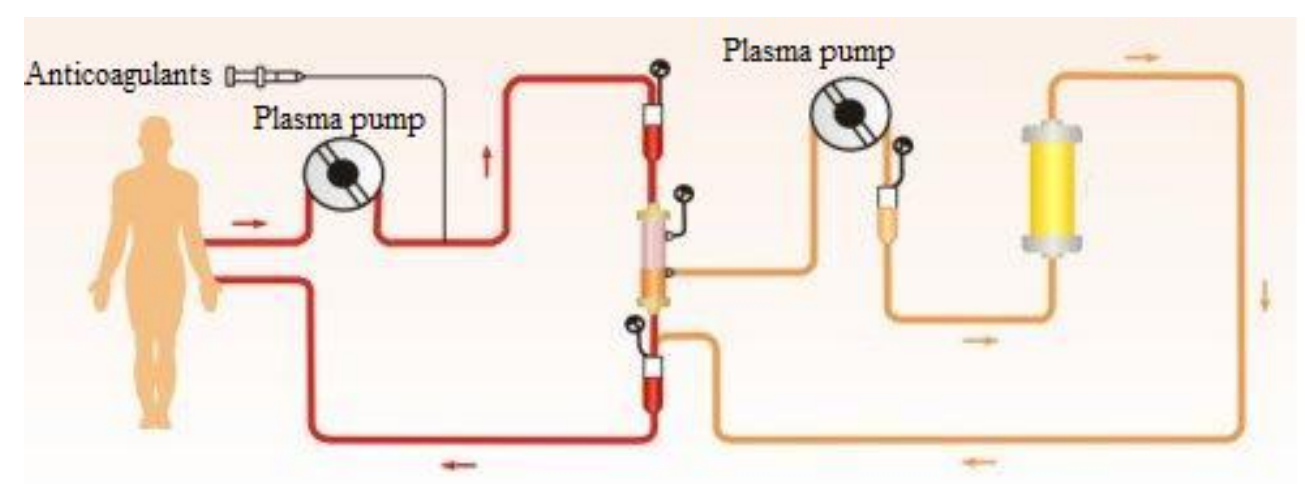

Figure 1. Blood purification schematic diagram

Hemodialysis. Department of Hemodialysis patients blood dialyzer is introduced, the use of semi-permeable membrane on both sides of the solute concentration difference by permeation, diffusion and ultrafiltration, to clear metabolites and toxic substances, correct water and electrolyte balance disorders purposes. Commonly used methods (1) arteriovenous reserved intubation. Generally used in the dorsal medial malleolus artery and saphenous vein catheterization; we can use 
expansionary catheter Seldinger puncture femoral artery and vein. In acute poisoning or emergency dialysis in acute renal failure. (2) arterial and venous fistula. Choice of the radial artery and accompanying veins of the head, with two silicone tubes were inserted into the move, to the heart side, the line outside the skin connected vein, formed in vitro shunt. For acute and chronic renal failure and the need for long-term dialysis. (3) arterial and venous fistula. Choice of the radial artery and its accompanying vein as side to side or end to side anastomosis; can also be used titanium wheel nail (pore size of $2.0 \sim 2.5 \mathrm{~mm}$ ) line anastomosis. After the agreement two weeks to work in the vein of the artery at the puncture for hemodialysis. Suitable for long-term dialysis. Under (4) subclavian vein catheterization. Under the double-lumen catheter is inserted into the subclavian vein, the blood side of the hole through the outer tube sucked out, flowing through the dialyzer, and then by the inner tube reinfusion in vivo.

Hemofiltration. HF is an analog device in accordance with the glomerular filtration function and design. HF by a hemofiltration device, a blood pump, a suction device consisting of three parts. (1). Establish arteriovenous passage and heparin method: with hemodialysis. (2) hemofiltration means: common polyacrylonitrile film multilayer small flat strainer, polysulfone hollow fiber membrane filtration, polymethyl methacrylate membrane filtration device and the like. (3). The end of the patient's arterial and venous blood filtration device, respectively arteriovenous pipeline connection, rely on the blood pump and filtration devices vein conduit clamps enable filtering the blood side produced 13.33 $26.66 \mathrm{kpa}(100 \sim 200 \mathrm{mmHg})$ positive, negative adjustment pressure device, the negative pressure reaches $26.66 \mathrm{kpa}$, you can get $60 \sim 100 \mathrm{ml} / \mathrm{min}$ filtrate was added at the same time replacement fluid. As per request the removal of the body $1000 \mathrm{ml}$ liquid is filtered out by subtracting the total amount of liquid $1000 \mathrm{ml}$, is the substitution of liquid input. (4). The replacement fluid composition and input methods: $\mathrm{Na}+140 \mathrm{mmol} / \mathrm{L}, \mathrm{k}+2.0 \mathrm{mmol} / \mathrm{L}, \mathrm{Ca}++1.85 \mathrm{mmol} / \mathrm{L}, \mathrm{Mg}++0.75 \sim 1.0 \mathrm{mmol} / \mathrm{L}$, Cl-105 110mmol / L, lactate $33.75 \mathrm{mmol} / \mathrm{L}$ dubbed. Can be input in the strainer arterial duct (before dilution type) or intravenous line input (diluted type).

Hemoperfusion. HP is introducing the arterial blood storage hemoperfusion adsorption material means by which to make contact with the blood of toxins, metabolic products adsorbed and purified, and then come back to lose the body. (1). The establishment of arterial and venous access and heparin method: with hemodialysis. (2). hemoperfusion device consists perfusion tank, adsorbents, microcapsule film. Clinical mainly albumin collodion wrapped activated carbon, charcoal and acrylic hydrogel wrapped acetate fiber wrapped activated carbon. Activated carbon is usually 8-14 purpose of coconut shell charcoal. (3). The patient's artery and vein are connected with arterial and venous blood conduit perfusion device, utilizing the blood pump to maintain blood flow $200 \mathrm{ml} / \mathrm{min}$ or so. Daily or every other day, every 2 to 3 hours, until the clinical symptoms improved.

Replacement Therapy. PE Department will be introduced into the patient's blood plasma exchange means the separated plasma was discarded, and to pay back a certain amount of plasma, in order to clear the plasma of patients with antibodies to activate the immune response of the media and immune complexes. (1). Establish vascular access and heparin method, with hemodialysis. (2). The plasma separation device: use of cellulose acetate film, poly methyl methacrylate film or a polysulfone membrane made of hollow fiber type separator. Membrane area of $0.4 \sim 0.6 \mathrm{~m}^{2}$, pore diameter $0.2 \sim$ $0.6 \mu \mathrm{m}$, the maximum cut off molecular weight of 300 daltons. (3). The patient's artery and vein, respectively, the plasma separator artery and vein pipe connections, adjust the speed of the blood pump with negative pressure, maintain blood flow $200 \mathrm{ml} / \mathrm{min}$, the ultrafiltration control plasma volume $30 \sim$ $60 \mathrm{ml} / \mathrm{min}$, means time is 90 120minutes, 2 times/week, each time the total amount of ultrafiltration to about 4 liters of plasma. From plasma filtration device venous transfusion $4 \%$ human albumin Ringer 3.8 liters (or $20 \%$ albumin $400 \sim 800 \mathrm{ml}$, the rest of the compound sodium chloride solution).

Peritoneal Dialysis. Peritoneal dialysis is peritoneal use as a semi-permeable membrane, the membrane according to the Southern and more balance principle, the prepared dialysate catheter poured into the peritoneal cavity of the patient, so that both sides of the concentration of the solute in the presence of a gradient of the difference between the peritoneum, the high concentration side low 
solute concentration to the side movement (diffusion); water from the low permeability (infiltration) hypertonic side to side movement. Constantly replaced by peritoneal dialysis solution in order to achieve clear metabolic products, toxic substances, and to correct water and electrolyte balance disorders purposes [5].

1. Peritoneal dialysis select (1) emergency peritoneal dialysis. In the short term for all day continuous dialysis. As more acute renal failure and acute poisoning rescue measures. (2) intermittent peritoneal dialysis. 5 to 7 days a week dialysis, dialysate daily $6000 \sim 10000 \mathrm{ml}$, four to eight times the input intraperitoneal, indwelling each 1 to 2 hours, 10 to 12 hours daily dialysis. For chronic renal failure with marked fluid retention persons. (3) continuous ambulatory peritoneal dialysis (CAPD). 5 to 7 days a week hemodialysis, daily dialysis 4-5 times, each time with dialysate $1500 \sim 2000 \mathrm{ml}$, enter the abdominal cavity, replace every 3 to 4 hours at night 1 indwelling intraperitoneal 10 to 12 hours. After peritoneal dialysis fluid poured into the clamping infusion tube, and the original fold bags containing dialysate into the waist pocket, put the liquid taken out, placed in the lower, so that dialysis fluid from the abdominal cavity through a peritoneal dialysis effluent pipe and then back to new peritoneal dialysis fluid bags. Patients in the ambulatory dialysis, patients can move freely. (4) continuous cycling peritoneal dialysis (CCPD). System using a computer program-controlled automatic cycling peritoneal dialysis machine. Patients with sleep at night, intraperitoneal indwelling peritoneal dialysis tube end connected with automatic cycling peritoneal dialysis machine, with 6 to 8 liters dialysate continuous dialysis 9 to 10 hours, early morning intraperitoneal memory remaining 21 dialysate from the machine, the entire During the day (10 to 14 hours) without replacing the dialysis fluid, patients can move freely during the day.

2. The peritoneal dialysis tube commonly used single hair sets (cuff), double sets of hair and hairless, three sets of silicone rubber peritoneal dialysis tube.

3. Catheterization $1 / 3$ puncture trocar at the navel and toe joint line, and then through the trocar tube into the peritoneal dialysis rectal bladder fossa, or surgical incision layered peritoneum, the peritoneal dialysis tube inserted rectovesical nest, to dialysis. Chronic renal failure and need for long-term peritoneal dialysis, could mainly as a tunnel in the abdominal wall, and with a hairy sleeve peritoneal dialysis tube tunnel piercing through the outer skin, to help fixed.

4. Dialysate dialysate formulation can be temporary or self-configured using a commercially available dialysate. Temporary dialysate formulations: $5 \%$ glucose solution $500 \mathrm{ml}$, saline $1000 \mathrm{ml}, 5 \%$ sodium bicarbonate $100 \mathrm{ml}, 5 \%$ calcium chloride $12 \mathrm{ml}$, osmotic $359.4 \mathrm{mmol} / \mathrm{L}$.

\section{Traditional Chinese Medicine}

Dachengqitang from the "Treatise on" therapeutic "Pi, full, dry, solid, hard," the Yangming Fushi as the ancient physicians respected. In the various versions of "prescriptions" textbook, Dachengqitang were included devaluation prescription purgative agent, thinks that the only product of thermal Jun, told Yangming Fushi, hot junction bypass, spasm disease. Dachengqitang syndrome prevalent: hot flashes delirium, hand, foot and was moved to sweating, frequent vector gas, constipation, abdominal $\mathrm{Fu}$ Man pain refused to press, tongue from the thorn sallow, dry cracked into a charred, slippery pulse Shen Chi or powerful. Lee or below water, foul smell unpleasant, umbilical abdominal pain, according to a block of the hard, hot Jue, high fever coma, bayan throw feet, cold drink irritability, constipation unreasonable; or see lockjaw, tetany, angle arch anti-sheets, lockjaw Kuwana tooth.

Dachengqitang of herbs rhubarb bitter cold, longer than purging fire detoxification, cold serum blood stasis, stop bleeding and has been used in a variety of severe treatment. Find rhubarb rhizomes contain rhein, emodin, rhubarb phenol, aloe emodin ether, free anthraquinone derivatives in modern research, no diarrhea induced. Glucoside and the other containing the above substances sennosides A, $\mathrm{B}$, C, etc. in combination form anthraquinone derivatives, has caused diarrhea effect. Further still contains tannin and so on. Rhubarb rhizomes and root have lower heat diarrhea, blood and broken product, anti-bacterial anti-inflammatory effect. Students with a steep prescription, concocted after use is a slow prescription. Then fried carbon can be used to stop bleeding. Taking a small dose of a 
stomach, convergence effect. Also rhubarb contains anthraquinone glycosides, it was yellow, laxative effect. And containing approximately $40 \%$ of calcium oxalate, so much grit. There are other ingredients emodin, gums, resins, Rhein, purgative butter and rhubarb with a convergence of tannic acid. It has confirmed that rhubarb can protect intestinal epithelial cells mitochondrial respiratory chain function, protect the intestinal mucosal barrier, improve gastrointestinal mucosal blood flow, thereby improving the barrier function of the gastrointestinal tract. Mechanism of Rhubarb emodin may stimulate the intestinal tissue of 5-HT antelope cells to secrete serotonin 5- antelope activities strengthened and 5- antelope serotonin receptor involved in promoting the bowel secretion and intestinal tract contraction of. Li Feng and other studies suggest that moderating effect on colon aquaporin rhubarb is rhubarb purgative effect reasons Dachengqitang gastrointestinal poison not only clear effect on the gastrointestinal tract itself or protection. Gastrointestinal tract is the body's largest library of bacteria and toxins, gastrointestinal mucosa is an important barrier against bacterial invasion and are highly susceptible to damage. Within Dachengqitang vitro directly inactivate toxins, the prevention of intestinal infection play a role, while serious infections reduce proinflammatory cytokines increased intestinal mucosal barrier protection, control of bacterial translocation, decrease endotoxin release, controlled gut-derived sepsis. In addition, Dachengqitang can lysosomal membrane stability and prevent the hydrolysis enzyme digestion and destruction of mitochondria.

\section{Conclusion}

Blood purification process, changes in plasma concentrations of poison depends on three factors: the poison of spontaneous clearance, clearing blood purification thereof, or poison tissue extracellular space or move within the blood cells from the body's deep. Ideally suited blood purification is to increase the blood concentration of toxic discharge of making changes, so that the target organ and tissue cells toxicant concentration is reduced, thereby shortening the duration of the patient cured, but in fact only a few poison comply with this condition. Large volume of distribution, with high affinity organizations toxicant concentration in the blood is very low, less poison in the blood affect tissue concentrations of toxicants through blood purification cleared.

\section{References}

[1] Wang Zhigang.Net blood chemistry (2nd edition) [M] . Beijing Science and Technology Press. 2003: p346-348.

[2] Hu Xiaodong. Application and evaluation of hemoperfusion in the treatment of paraquat poisoning [J]. Occupational Health and Damage. 2003, 18 (2): 131-133.

[3] Branch of the Chinese Medical Association of Emergency Physicians. Diagnosis and treatment of acute paraquat poisoning expert consensus (2013) [J]. Chinese Critical Care Medicine, 2013, 33 (6): 236-240.

[4] Zhang Zhengwei, Ying Xiangdong, Ruan Yanjun. Xuebijing injection in the treatment of acute paraquat poisoning experiment [J].Journal of Toxicology. 2007, 21 (2): 105-108.

[5] Xu Minghua, Zhang Haisong, Liang Wei. Paraquat poisoning of oxygen free radicals and changes in blood perfusion effect [J]. Chinese Journal of Modern Medicine. 2008,10 (2): 4-5 